\title{
Secondary findings in exome slices, virtual panels, and anticipatory sequencing
}

\author{
Leslie G. Biesecker, MD, FACMG ${ }^{1}$
}

The terms "virtual panels" and "exome slices" or "custom panel" describe the practice of marketing, describing, or making available a test as comprising a modest size set of genes (e.g., an ataxia gene panel), when in fact a much larger set of genes was actually interrogated (e.g., an exome, the socalled Mendeliome, or a single superset of genes comprising all gene components of multiple, individually marketed panel tests). In this model, the laboratory need only invest in a single molecular biology process and quality assurance process and can then parse this single data set into multiple testing products using only bioinformatics tools. A second, related practice is the model of sequencing individuals (at a low cost, or below cost) to make available an exome or genome data set that can be incrementally reinterrogated over time by patient/customer requests for subsets of results (whereby the costs of the initial sequencing can be recovered). For lack of a better term, I will describe this as "anticipatory sequencing." While the initial appeal of these approaches is obvious, these practices are problematic with respect to secondary findings.

The American College of Medical Genetics and Genomics (ACMG) has promulgated ${ }^{1}$ and refined over time ${ }^{2,} 3$ recommendations for secondary findings, which did not precisely parse this ambiguity when they stated "...the laboratory (should) evaluate the sequence data on these genes that are generated in the course of routine clinical sequencing." These recommendations espoused the principle that if a pathogenic variant in one of the listed genes was detected by the underlying molecular processes of exome or genome sequencing, it should be returned. Equally important is to recognize that they did not recommend or require that any of the molecular biology processes for an exome or genome sequencing test need to be modified to ensure that all 59 of these genes are adequately sequenced by the exome or genome assay. The idea was that if the sequence data were generated, the policy applies, and the variants that are detected are subject to the secondary findings recommendations. If part or all of any of the 59 genes are not adequately sequenced by the exome or genome assay, that unsequenced portion of the genome obligates no efforts by the laboratory to generate such data another way. I am proposing no change in these concepts regarding secondary findings. I simply interpret them to mean that what is sequenced is subject to the recommendation, and what is not sequenced is not subject to the recommendation.

As noted above, the ACMG recommendations considered only exome and genome sequencing but did not consider virtual panels, exome slides, or anticipatory sequencing and therefore raise important questions about the applicability of the recommendations in these three scenarios and more fundamentally, the concept of latency of genomic variant data. Here, I use the following definition of the word latent to mean "Existing or present but concealed or inactive," which is to say that certain genomic variants in the patient's data have been detected by the sequencing, but are being treated as though they have not. Latency is critical-the ACMG policy seeks to address variants that are identified, not to specify what genes should be interrogated. The current practice of some laboratories performing exome slices, virtual panels, and anticipatory sequencing is to not offer secondary findings on the now 59 genes recommended for secondary findings returned to patients undergoing these types of tests, when these genes are included in the molecular testing processes. ${ }^{3}$ I suggest that these practices are inconsistent with the ACMG recommendations. Some other professional bodies have generally endorsed the ACMG approach to secondary findings, ${ }^{4}$ but are ambiguous or silent on the issue of virtual panels, exome slices, and anticipatory sequencing, which further supports the need for a discussion of the issue.

There are several arguments that support the offering of secondary findings from virtual panels, exome slices, or anticipatory sequencing, when one or more genes from the ACMG 59 list is interrogated by the underlying molecular processes. The first is conceptual. In all three of these testing modes, there is no conceptual difference between those practices as compared with an exome or genome sequence for an indication that does not overlap with genes on the secondary findings gene list (e.g., autism). In exome testing,

\footnotetext{
${ }^{1}$ Medical Genomics and Metabolic Genetics Branch, National Human Genome Research Institute, National Institutes of Health, Bethesda, Maryland, USA. Correspondence: Leslie G. Biesecker (lesb@mail.nih.gov)
} 
the laboratory is setting out to interrogate a set of genes related to autism, and in the process of doing that, the exome result is subject to secondary findings recommendations for return (absent an opt-out), even though these latter genes are unrelated to autism. Some laboratories offer virtual panels that are based on an underlying exome assay-they are just choosing to call an exome a virtual autism panel. Whether one describes that process as a clinical exome for autism or an autism virtual panel or exome slice is arbitrary. I suggest that the ACMG secondary findings recommendations apply to virtual panels that are based on an exome or genome platform.

A second issue is that a number of laboratories that offer virtual panels include interrogation of a number of genes that are on the ACMG secondary findings gene list among one or more of their panels. For example, a laboratory may offer a virtual panel for epilepsy and virtual panels for inherited cancer and cardiomyopathy. In one hypothetical, all three of these virtual panels are in fact implemented as a single, multigene capture and sequencing process that includes epilepsy, cardiomyopathy, and cancer-related genes, and the cardiac and cancer virtual panel gene lists include one or more genes from the ACMG 59 list and the epilepsy list includes none. In this hypothetical, the sequence data generated for all three of these virtual panels is the same, but only one of the three sets of genes is interpreted and returned for each virtual panel. I argue here that the ACMG secondary findings policy applies to all three virtual panels for what portion of the ACMG 59 gene list genes are on that underlying molecular biology assay. When the epilepsy virtual panel is ordered, the subset of cardiac and cancer gene list that overlaps with the ACMG 59 list should be offered for return as secondary findings. If the cancer virtual panel, the cardiomyopathy virtual panel genes that are on the ACMG 59 list should be returned. In a different hypothetical, these three panels instead have separate molecular processes, that is, each of the three panels only includes molecular processes to capture and sequence either epilepsy genes, or cardiac genes, or cancer genes. In this case, the ACMG secondary findings do not apply-because when the epilepsy panel is run on a given patient, the cardiomyopathy and cancer genes have not been sequenced, so there are no secondary findings to report. When the cardiomyopathy panel is ordered, the ACMG policy does not apply because that panel is presumably being done for the purpose of evaluating cardiomyopathy, so the findings are all primary, not secondary. As discussed above, this follows the previously articulated ACMG recommendations-if one or more of the 59 genes is sequenced, the secondary findings recommendations apply. If none are sequenced, the recommendations do not apply and the laboratory is not obligated to generate sequence data for any of those 59 genes.

This is also an issue for anticipatory sequencing services that are connected to downstream or supplemental interpretations (which may be purchased as a clinical service, perhaps using an online app or interface with the testing laboratory that allows a patient/customer to request interpretations of additional genes). If their processes are connected to an interpretation module for hereditary cancer or cardiomyopathy, they are demonstrating the capability to do these interpretations. Based on the existence of these capabilities, it would seem disingenuous for the laboratories to represent that they do not have such capabilities or processes in place. I interpret the ACMG secondary findings recommendations to apply to anticipatory sequencing and recommend that secondary findings should be offered for all persons who undergo such testing as part of the initial interpretation service.

The final issue is the most important, and that is the viewpoint of patients and the public. From their perspectives, the highly technical, arcane, and obscure issues argued above are likely to be irrelevant and unimportant. We should expect that the patient who has suffered harm from the lack of an opportunity to receive secondary findings will not be comforted or assuaged by technical arguments, when it is in fact the case that the variant was, in fact, actually detected in the assay and could readily have been evaluated. The broad support of the public for the biomedical enterprise depends on their perception that the enterprise is overall acting in their interests. For our field to pretend that secondary variants do not exist, when they in fact do, undermines that trust.

Some have explicitly proposed the opposite of what I am arguing here. This is the notion that if variants in secondary findings genes are generated in the molecular biologic processes but subsequently blocked or filtered using informatics, then the problem is resolved. An example of this is the following: "Variant filtering to selectively assess only those variants in established disease genes that may be related to a patient's condition (phenotype-driven) is an effective way to minimize interpretation requirements, while maintaining test effectiveness. This method also reduces the chances of unintentionally identifying potential disease-associated variants unrelated to the primary indication (incidental findings)". ${ }^{5}$ Another is "...some favored restricting analysis to virtual gene panels, intentionally minimizing nonprimary findings". 6 To develop a policy precluding the return of secondary findings is justifiable if one argues that they are not adequately predictive, or that they are not cost effective, or that professionals choose to override the widely expressed preference of patients to receive them, but it is not justifiable to pretend they are not there, latent within the data.

I propose that the ACMG secondary findings recommendations apply to exome slices, virtual panels, and anticipatory sequencing when secondary findings genes are included in the upstream molecular biologic processes and are identifiable as valid genotypes through routine informatics processes used for germline sequencing. Laboratories that offer these services should disclose to patients/ customers that secondary findings from such assays are a possibility, and provide a pathway or mechanism for the patient/customer to receive such findings and the possibility of opting out, in conformance with the ACMG secondary findings recommendations. 


\section{ACKNOWLEDGEMENTS}

The opinions expressed here are those of the author and do not necessarily represent any policy or position of any institution or other organization to which he is affiliated.

\section{FUNDING}

The author is supported by the Intramural Research Program of the National Human Genome Research Institute, grant HG200387 04.

\section{DISCLOSURE}

The author is an unpaid consultant to the Illumina Corp and receives royalties from Genentech, Inc. The author receives salary and grant support from the National Institutes of Health.

\section{REFERENCES}

1. Green RC, Berg JS, Grody WW, et al. ACMG recommendations for reporting of incidental findings in clinical exome and genome sequencing. Genet Med. 2013;15:565-74.

2. ACMG Board of Directors. ACMG policy statement: updated recommendations regarding analysis and reporting of secondary findings in clinical genome-scale sequencing. Genet Med. 2015;17:68-69.

3. Kalia SS, Adelman K, Bale SJ, et al. Recommendations for reporting of secondary findings in clinical exome and genome sequencing, 2016 update (ACMG SFv2.0): a policy statement of the American College of Medical Genetics and Genomics. Genet Med. 2017;19:249-55.

4. Robson ME, Bradbury AR, Arun B, et al. American Society of Clinical Oncology Policy Statement Update: Genetic and Genomic Testing for Cancer Susceptibility. J Clin Oncol. 2015;33:3660-7.

5. Kernohan KD, Hartley T, Alirezaie N, et al. Evaluation of exome filtering techniques for the analysis of clinically relevant genes. Hum Mutat. 2018;39:197-201.

6. Ormondroyd E, Mackley MP, Blair E et al. "Not pathogenic until proven otherwise": perspectives of UK clinical genomics professionals toward secondary findings in context of a Genomic Medicine Multidisciplinary Team and the 100,000 Genomes Project. Genet Med 2018;20:320-8 